\title{
Experiences of Fast Queue health care users in primary health care facilities in eThekwini district, South Africa
}

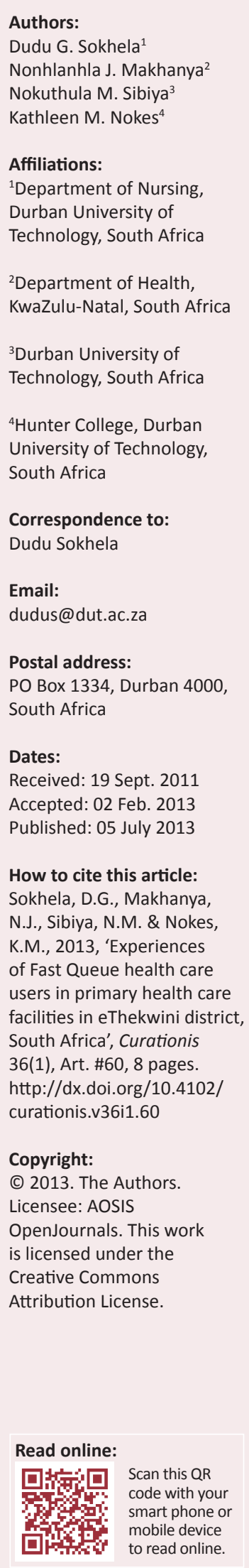

Background: Comprehensive Primary Health Care (PHC), based on the principles of accessibility, availability, affordability, equity and acceptability, was introduced in South Africa to address inequalities in health service provision. Whilst the Fast Queue was instrumental in the promotion of access to health care, a major goal of the PHC approach, facilities were not prepared for the sudden influx of clients. Increased access resulted in long waiting times and queues contributing to dissatisfaction with the service which could lead to missed appointments and non-compliance with established treatment plans.

Objectives: Firstly to describe the experiences of clients using the Fast Queue strategy to access routine healthcare services and secondly, to determine how the clients' experiences led to satisfaction or dissatisfaction with the Fast Queue service.

Method: A descriptive qualitative survey using content analysis explored the experiences of the Fast Queue users in a PHC setting. Setting was first identified based on greatest number using the Fast Queue and geographic diversity and then a convenience sample of health care users of the Fast Queue were sampled individually along with one focus group of users who accessed the Queue monthly for medication refills. The same interview guide questions were used for both individual interviews and the one focus group discussion. Five clinics with the highest number of attendees during a three month period and a total of 83 health care users of the Fast Queue were interviewed. The average participant was female, 31 years old, single and unemployed.

Results: Two themes with sub-themes emerged: health care user flow and communication, which highlights both satisfaction and dissatisfaction with the fast queue and queue marshals, could assist in directing users to the respective queues, reduce waiting time and keep users satisfied with the use of sign posts where there is a lack of human resources.

Conclusion: Effective health communication strategies contribute to positive experiences by health care users and these can be effected by: (1) involvement of health care providers in planning the construction of health facilities to give input about patient flow, infection prevention and control and provision of privacy, (2) effective complaints mechanisms for users to ensure that complaints are followed up and (3)encouraging users to arrive at the facility throughout the day, rather than the present practice where all users arrive at the clinic early in the morning.

\section{Introduction}

The South African government reorganised healthcare and created a Comprehensive Primary Health Care System (PHC) using the World Health Organization (WHO) principles (WHO 1978) of accessibility, availability, affordability, equity and acceptability. Sibiya (2009) described integrated primary health care as a strategy that increases health service utilisation by increasing accessibility and availability of all health services at the PHC level. The way that organisations serve clients who are waiting to be seen, impacts on the clients' perception of the organisation. Queue management is about ensuring fairness and demonstrating to clients that they are waiting in a planned environment, whilst reassuring them that they will be attended to timeously (Qmatic. com 2005). Clients want to know that their time is valued. Patient flow and actively searching for bottlenecks is necessary to ensure that patients are processed through to the fastest or shortest queue at any given time. The Fast Queue strategy was implemented to resolve challenges in a range of health care services so that patients coming for a brief, routine consultation would not be kept unnecessarily in the PHC setting for long periods.

The WHO estimates that one-third of the 40 million people living with HIV and AIDS worldwide are co-infected with tuberculosis (TB), one of the most common opportunistic infections. South Africa has one of the highest estimated TB rates in the world, ranking fourth amongst the $22 \mathrm{WHO}$ determined high-burden countries, with an estimated 461,000 new cases reported each year. The 
South African TB and HIV co-infection rate is also high, with approximately $55 \%$ of $\mathrm{TB}$ patients also testing positive for HIV. Further exacerbating the problem is the existence of drug-resistant $\mathrm{TB}$, often caused by non-adherence to drug regimens (CDC 2011). One of the challenges has been that the changes in the healthcare system planned by the postapartheid government, could not anticipate the great influx of patients infected with tuberculosis and HIV who would require services in their local community.

\section{Problem statement}

Whilst the Fast Queue strategy has been instrumental in promoting access to health care services, a major goal of the PHC approach; the influx of patients as experienced by the researcher, has had adverse effects on health care delivery, with queues growing longer and patients waiting many hours for service. Although the Fast Queue strategy was introduced in 2001, there is no evidence of any systematic evaluation by South African researchers, hence the need for this study.

\section{Background}

Poorly staffed health care facilities in South Africa have been faced with the challenge of treating persons with complex health problems who present at the PHC level. Waiting times due to long queues had increased in public health clinics, which prompted the Gauteng Member of the Executive Committee for Health and Social Development, to announce that waiting times would be a thing of the past and that additional queue marshals would be introduced to assist outpatients (Mahlangu 2009:1). The Fast Queue strategy is the service for patients who need short consultations and have been seen previously in a hospital, community health centre or another primary health care facility and referred for ongoing treatment (Department of Health 2001).

Chronically ill adults, older adults and children could be referred to the Fast Queue if they had been assessed previously either at a Comprehensive Health Centre (CHC) or PHC clinic. Services provided to in the fast queue include: follow up issuing of prescribed treatment according to protocols along with routine procedures, including assessment of vital signs and basic physiological assessment parameters such as blood pressure, body mass index, heart and lung functioning, and blood glucose levels. Patients who further qualify for the Fast Queue service include those who need follow up monitoring of complications; to identify and refer people with disabilities; patients who need provision of explanation and education on treatment and general health education in group or individual format as well as for the booking of follow up visits. In addition, Fast Queue services include the treatment and follow up of patients and families of patients with mental diseases; dispensing and monitoring of medication for acute conditions for a limited period according to protocol; identification and referral for periodic reassessment; screening, counselling and referral for drug abuse, sexual abuse, child abuse and other crisis events.
Services for children in the Fast Queue include: growth monitoring and immunisation; counselling of caretakers; rheumatic heart disease prophylaxis; counselling of parents regarding the prevention, impact and follow up related to teenage pregnancies including; providing reports to the school, social welfare services or other service providers on request, and/or in agreement with parent or legal guardian. Other services included in the Fast Queue are for persons with special needs such as working clients who can visit the clinic at times suited to their working hours or patients who need to be attended to very early, before other services start - such as patients needing family planning methods, daily Directly Observed Treatment Short Course (DOTS), chronic patient medicine collection, immunisations, and other agreed upon services such as dispensing of sunscreen for people with albinism (Department of Health 2001:28). Since a significant number of chronically ill patients who visit the primary health care facility can access the Fast Queue service, it is essential that services are continuously evaluated and patient perceptions and experiences are assessed.

\section{Aims of the study}

To describe the experiences of the users of the Fast Queue in order to better understand how to more effectively implement the Fast Queue strategy and thus, improve health care delivery and enhance user satisfaction.

\section{Objectives}

To describe the experiences of the Fast Queue strategy users and to determine the level of satisfaction of users with Fast Queue strategy.

\section{Purpose}

This study explored experiences of health care users of the Fast Queue strategy to learn about their perceptions and experiences with this service.

\section{Operational Definitions}

PHC facilities are clinics within communities which are the first contact for health care.

Health care users are all people; whether sick or well, who visit the PHC facility with the purpose of seeking health care.

\section{Health care providers are nurses working in the Primary Health Care facility.}

Significance: The results of the study will assist in decision making related to patient flow management, in order to reduce patient waiting times which in turn will improve access to healthcare services and reduce overcrowding. With improved patient flow, health care providers may work more effectively and user satisfaction may increase. The study will inform policy makers who can address unnecessary long waiting times in the comprehensive health care service package for South Africa. 


\section{Theoretical Framework}

The Kano Model of Customer Satisfaction (Kano 1984:1) is a quality management and marketing technique that was used to guide the study (see Figure1). The Kano model of customer satisfaction classifies product attributes based on how they are perceived by customers and the effect they have on customer satisfaction. According to this model, there are six attributes, namely; basic or threshold, performance, excitement, neutral, questionable and reverse attributes however, only the basic or threshold attribute will be addressed in this article. This attribute is compulsory for any product and should not be omitted at any given time because absence results in customer dissatisfaction. One of the essentials of the basic or threshold attribute is the presence of qualified and highly skilled health care providers at the health facilities, who can make expert decisions about health care needs. Patients perceive that they are in good hands, the providers knows what they are doing and do so with agility. This will facilitate quality care and avoid delays, thus shortening patient waiting time and increasing satisfaction.

\section{Literature Review}

Customers are satisfied if the quality of goods or services of an organisation meet the specified expected requirements (Oakland 2003:4). Waiting times relate directly and form a significant component of patient satisfaction (Eilers 2004:1). Thompson and Yarnold (1995:662) found that patients were least satisfied when the waiting time was longer than expected; they were relatively satisfied when time was perceived as equal to their expectation and they were highly satisfied if the waiting time was shorter than expected. Ajayi (2002:1-7) concluded that if patients perceive that the time they have to wait is used constructively, the time was well spent and they have gained from it. Setty (2004:2) identified important principles of effective client flow in reproductive health and family planning settings, namely; balancing client load with client flow. Improving client flow can help shorten patient waiting time and thus, increase the number of patients a provider sees daily. Effectively using signs, posted

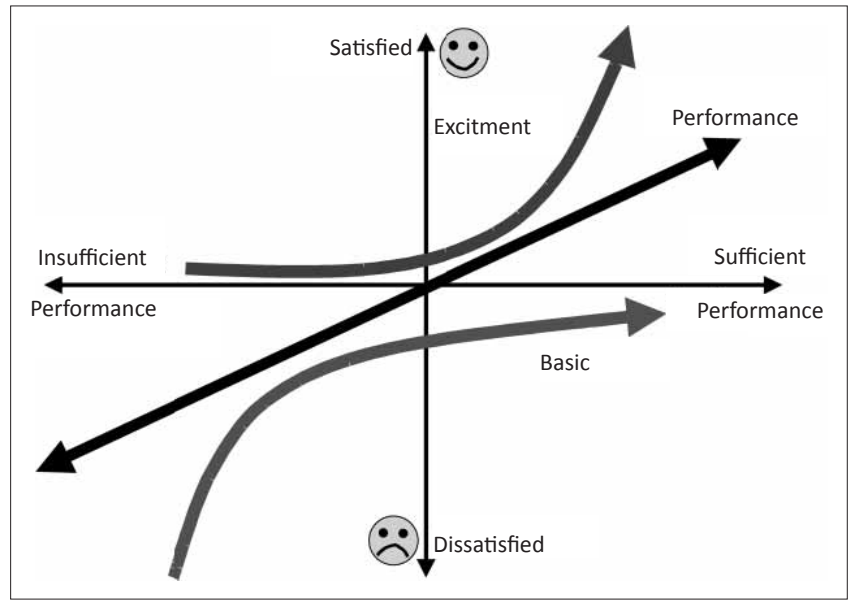

Source: Kano's Customer Satisfaction Model (1984)

FIGURE 1: Customer satisfaction model. or written instructions and simplified and clear directions can assist the clients' time to find service provision areas on their own and move through the system more quickly.

\section{Research method and design Design}

A descriptive qualitative survey using content analysis (Burns \& Grove 1999) was used to explore the experiences of the health care users served on the Fast Queue.

\section{Sampling}

In order to ensure geographic diversity, a two stage sampling plan was used. To identify the PHC setting in the three health sub-districts (south, north and west sub districts of eThekwini municipality), facilities with the highest number of attendees seen over three months (October 2008 to March 2009) were chosen. Once the clinic was selected, health care users who were in the Fast Queue on the day of the research were purposively selected. Since one of the most frequent services provided by the Fast Queue is for chronically ill patients who come monthly to collect their medications, it was decided to hold one focus group with those health care users to gain insight into their unique experiences as regular users of the Fast Queue strategy.

\section{Data collection method}

After informed consent was obtained, the same interview guide questions were used for both individual and the one focus group discussion. The researcher conducted the interviews in the languages the participants were comfortable with, mainly isiZulu and English. Responses were audio taped and transcribed in English by the researcher. Data was transcribed in the languages that interviews were done in, and those done in isiZulu were translated into English.

\section{Context of the study}

The study was conducted in the PHC facilities of eThekwini Municipality excluding mobile units. The eThekwini Municipality is situated within eThekwini district, which is one of 11 districts in the province of KwaZulu-Natal in South Africa. The municipality was formulated following the December 2000 election and is a product of seven municipalities that were amalgamated into one. It is divided into three sub-districts namely South, North and West. The Municipality has 77 PHC facilities, and 15 mobile units, with $1 \mathrm{CHC}$ shared between the municipality and the provincial department of health. The catchment population ratio per clinic is 1:22570, which is above the national norm of 1:15000 (eThekwini municipality 2006/2007).

\section{Data Analysis}

Data analysis occurred in three stages namely; description, analysis and interpretation. In the description phase the researcher listened and re-listened to the interviews from the 
audio tape recorder. The researcher also read and re-read the transcripts to become immersed in the data with the aim to interpreting it to find meaning and make sense of it. In the analysis phase, themes and sub-themes were identified from the raw data to establish patterns and these were used to interpret data. The researcher interpreted the data according to her understanding using; themes and sub-themes identified during the analysis phase, and supported her interpretation with the use of excerpts from the interviews to eliminate subjectivity and bias. The Kano model was used to guide identification of themes into specific categories.

\section{Results}

Five clinics, with the highest number of attendees during a three month period, and a total of 83 health care users of the Fast Queue were interviewed. The average participant was female, 31 years old, single, and unemployed.

According to Kano, basic and threshold attributes are foundational. Positive experiences with the Fast Queue resulted in client satisfaction whilst negative experiences resulted in dissatisfaction. Two main themes: health care user flow with four sub-themes and communication with two sub-themes emerged.

\section{Theme 1: Health Care User Flow}

Good clinic organisation eliminated bottlenecks and effective health care user flow enhanced the work process and increased satisfaction. Sub-themes that emerged related to health care user flow and included: floor space, staffing patterns, waiting time, and workload.

\section{Floor space}

The clinic floor plan and structure had a direct bearing on user flow. Health care users shared their experiences in a small clinic which resulted in delays and long waiting times. Some users reported that the Fast Queue enabled quick attendance to an extent that allowed them to keep to their normal daily schedule. Mothers of young babies liked the fact that they were seated away from the sick people, thus protecting their children from possible infections.

'This queue is very fast because when I arrived here there were many of us and I thought I will leave late. When I came inside, there were many different queues that we were directed to. It was fast'. (Participant: 7, female, unemployed, 26 years)

'I am not sure. Maybe it is the load of work. This clinic used to be very full before it was moved to these new premises. Since moving to this new clinic the work seems lighter, but I think it is because this clinic is more spacious, there is more space for us to move and be separated into different specific queues'. (Participant: 22, female, unemployed, 32 years)

\section{Staffing Patterns}

Insufficient staffing contributed to dissatisfaction and resulted in increased waiting time. Although they were sympathetic, health care users still expected good and fast service. Staff shortages resulted in work starting late because of sorting out problems in other areas. This yielded high levels of dissatisfaction, which was expressed as follows:

'I am okay with it, but the staff is short, so the Fast Queue is not so fast. For an example: people with TB are not supposed to wait in the general waiting area, they are supposed to go straight to the nurses because other people can contract TB. Sometimes we are told there are only two or three nurses in the clinic.'(Participant: 17 , male, unemployed, 40 years)

'[I]t's just that they start late on some days. The nurses are few sometimes only two attending to patients. At the moment there is no one; I think she is at tea. Some can say they are neglecting their work'. (Participant: 18, male, unemployed, 47 years)

Whilst there is a shortage of staff, some health care users verbalised that there were more nurses than before 'and there are definitely more nurses now.'(Participant: 37, male, unemployed, 35 years)

\section{Waiting time}

Government health institutions are overcrowded, with long waiting times and long delays. Long waiting was mainly experienced during the process of registration and not in the fast queue. In the fast queue waiting time was minimal as expressed by users in these quotes:

'I was very happy because it saved me a lot of time; I am going to be able to go to class at $12 \mathrm{~h} 00$. I had even reported at school that I will be absent, but because it is organised and fast I am now going to be able to go to school'.(Participant: 47, female, university student, 20 years)

'There is no waiting after you have registered. The queue for babies is fast and I like it because you leave before the baby gets bored. It is very upsetting if you are in the clinic and the babies cry non-stop. With the fast queue it does not happen'. (Participant: 21, female, unemployed, 28 years)

Participants who experienced long waiting time had negative perceptions. A health participant, who had come for family planning, had this to say:

'Waiting makes people fed up and they can leave before they are attended to, and end up falling pregnant because maybe you are rushing to work, you can get fired for late coming. Therefore one may decide to leave if the queue is not fast'. (Participant: 53, female, 32 years)

Participants, who had brought babies for immunisation and waited long periods, expressed their experiences as:

'The queue was not fast at all. I waited very long to be seen with the baby, the nurses are slow. Though I cannot count the hours, but I waited long'.(Participant: 61, female, teacher, 33 years)

'The waiting is very tiring, especially if you have a baby and when you are pregnant, you come here in the morning, you are tired and stressed as well. They should come in earlier to open for us because we are standing from $05 \mathrm{~h} 30$ and they only come at $07 \mathrm{~h} 30$. If they can ask the guard to open for us'. (Participant: 30, female, unemployed, 27 years)

\section{Workload}

Increased workload was perceived as a factor contributing to long waiting times. Participants observed that on a particular day of the week the health care provider had a higher number 
of health care users because there were admissions from the hospital which increased the work load. The following quotes express the health care users' views:

'I cannot say, but I think it contributes to service delivery. It happens that, on Monday there are admissions which take long to open files for. I suggest that there is a way of attending to people coming to collect tablets only and not mix them with new admissions because that's what takes up time. Sometimes one is hungry because he knows he is only collecting tablets so it should not take long. That should be corrected. Dealing with medication only is very quick, and one does not even spend five minutes'. (Participant: 9, male, labourer, 38y years)

'It depends on days. Mondays are full because most people come in from hospital to start treatment and open files in the clinic. The queue is faster on Tuesday, Wednesday and Thursday because there are no admissions. Friday is also ok, no new admission'. (Participant: 11, male, unemployed, 40 years)

'The only problematic day is Monday. He also works hard for a long time that is why he loses his temper'. (Participant: 14, Female, domestic worker, 47 years)

\section{Theme 2: Communication}

Communication emerged as another theme that influenced satisfaction or dissatisfaction of participants in the Fast Queue. Data revealed that some participants experienced good communication with health care providers whilst others reported dissatisfaction with their exchanges. They stated that good communication meant good care and enhanced satisfaction with the Fast Queue and the clinic as a whole. Sub-themes that emerged related to communication, included: effective sharing of information and health care provider attitudes.

\section{Efficient sharing of information}

When health care users were informed, they reported understanding and acceptance of delays as highlighted in the following excerpts:

'I don't mind that there was a small problem which a nurse explained to me nicely because I thought I was going to go first since I was number one, but they explained that babies go first'. (Participant: 66, male, university student, 23 years)

'I commend the clerks, they are very fast. They kindly give you information and when they recognise a person that is in pain, on a wheelchair or a child, they take them first and help them. They explain about babies, I like that, it is very good. It's my first time here, but I like the way they work'. (Participant: 15, male, labourer, 37 years)

Other participants reported on their dissatisfaction with poor or no communication from the health care providers. Poor communication was perceived as poor staff attitude and was associated with dissatisfaction with the Fast Queue. This is how dissatisfaction was expressed:

'I am not satisfied with the service provision; the person who renders service does not know how to communicate with patients, because he is the provider, he needs to communicate effectively'. (Participant: 13, male, unemployed, 39 years)

'They sent me to the immunisation queue where I was sent back to weigh the baby, which I had not done when I went in for the baby's illness and I did not like that'. (Participant: 77, female, scholar, 19 years)

\section{Health care provider attitudes}

Health care provider attitudes also impacted on experiences with using the Fast Queue. Negative perceptions of staff attitudes affected evaluation of services and some said negative staff attitudes deterred them from visiting the clinic as expressed by the following quotes:

'Because with immunisation we had a problem before, the male who gave injections did not treat us well. The injection site would be swollen, he would not care even when you came back to complain. It is better now; this new male nurse treats babies well and plays with them. This other one used to just give an injection; that is all and call the next one. Sometimes if the baby cries, he would tell you to go and change the baby's nappy even if you had just changed the baby. It is better now'. (Participant: 39 , female, unemployed, 23 years)

'The nurses do not show that they care. Their faces tell you that they do not have the time for you. I was lucky my baby got an injection just before they went for tea. My sister, the hand cannot do what the face is not doing and the face cannot do what the heart is not doing. I feel that nurses should be taught how to work with people. This is a public service, we voted and we expect to be treated nicely. My baby is small; this is my second time here. They do their work but they do not have a way of interacting with the people. We are patients here but we are also human beings, they need to think about that'. (Participant: 27, female, unemployed, 28 years)

Positive experiences were noted as expressed by the following quotes:

'This guy looked at me and smiled saying and I said to myself: "[T]his is a pantsula, a guy from the township. I can see even in his step". We greeted each other and it was nice, I wish he would be there every day, but I could not do anything because I am not part of the administration, but I felt very comfortable with him'. (Participant 5, male, labourer, 39 years)

'I was happy, very happy. I think the attitude of nurses is changing towards patients and is changing for the better. I hope other clinics do the same'. (Participant: 47, female, domestic worker, 35 years)

\section{Ethical considerations}

The Durban University of Technology Ethics Committee approved the research and the eThekwini Municipality Research Committee granted permission to conduct the study. All participants were assured of voluntary participation; signed a written consent after reading and understanding the information letter. They were also informed that they could refrain from answering questions that they were not comfortable with and could withdraw at any stage of the study if they so wished. The assurance of total confidentiality at all times and that their participation would not compromise the way they would be treated in the facility, were conveyed to participants. They agreed that the findings and recommendations would be made available to the authorities and a copy would be kept in the municipal health library. 


\section{Trustworthiness}

Credibility was ensured through interviewing various participants from different PHC settings that used Fast Queue strategy. An audit trail was developed. Interviews were kept on audio compact disc, transcripts in the original language used in the interviews and a translated version, summaries of interviews, signed consent forms and correspondence with management. This was to enhance dependability. Confirmability was achieved by the use of direct quotes from the raw data to eliminate subjectivity and bias of the researcher. Transferability was achieved by providing sufficient descriptive data in the research report such that other researchers can test its applicability to other contexts.

\section{Discussion}

Two themes emerged: health care user flow and communication which could be consisted basic or threshold attributes and relate to their level of satisfaction or dissatisfaction with the Fast Queue. Results can be valuable for healthcare planners interested in improving client experiences with PHC services. Participants experienced higher levels of satisfaction when they perceived the Fast Queue to be fast and effective in delivering high quality service, especially in terms of user flow with four sub-themes (floor space, staffing pattern, waiting time and workload) and communication with two sub-themes (efficient sharing of information, and health care provider attitude). Good organisational skills by health care providers were important in user flow. Where health care providers were able to channel users in a logical manner, work progressed smoother and faster. Sending the users back and forth between services was a typical example of poor work organisation, which led to unnecessary delays and dissatisfaction. Fomundam and Herrmann (2007:1-15) noted that a patient may have to go through several nodes in order to be moved along for consultation, thus contributing to long waiting times. The Queuing Management System (Qmatic.com 2005) proposes that an active search for bottlenecks is necessary so that the patient is channelled through to the fastest or shortest queue at any given time.

The National PHC Facilities Survey conducted in 2003 by Health Systems Trust, revealed that waiting areas were adequate only in $48 \%$ of facilities, with lack of adequate seating being the most common deficiency. This is an indication that space is of utmost importance in health care facilities (Republic of South Africa 2004:29). Availability of space facilitated user flow since patients could be moved around and accommodated easily in the different queues. This was important since every user was able to be directed to the queue that provided necessary services without any delays and confusion because of lack of space. It also assisted in minimizing exposure to infectious illnesses including TB and health care users noted this and expressed their satisfaction ... This was especially noted with small babies who visited the well-baby clinic for routine assessments and immunisation. Even though in some facilities users had to be accommodated in separate buildings, they were still satisfied as long as consultations were completed quickly and comprehensively.

Shortage of staff was identified as another factor that resulted in slow queues and dissatisfaction. The introduction of free health services through the national health plan in 1996, to increase access to PHC and improve the health of the poor in South Africa, has had significant effects on staffing and work load, putting strain on the already poorly staffed facilities. Leatte, Shung-King and Monson (2006:51-56) support the statement that free health services have resulted in staff shortage, amongst other factors. Furthermore, the emergence of the HIV and AIDS pandemic and the down referral of stable chronic users from hospitals, are some of the factors that affected staffing in PHC (Hall 2001:5, Pendukeni 2004). Barron, Day, Loveday, and Monticelli (2005:9) suggested that the clinical workload per nurse should be 35 patients. In certain districts the patient load, however, reaches 92 patients per professional nurse per day (Barron et al. 2005:9). Hall (2001:6) found that the secrecy surrounding HIV and AIDS increased the nurses' workload with applying universal precautionary measures which take more time to administer. Not keeping the HIV results centrally contributed to increased workload. Another phenomenon observed by Hall (2001:6), is that patients shop around and visit several health service providers. This results in duplicating work for nurses. Caring for Patient with HIV-related illness or disease is demanding and time consuming. As more persons with HIV are able to access effective medications to treat their disease, acuity levels will decrease but the large numbers of HIV infected persons will continue to access the South African health care facilities well into the 21st century.

Oosthuizen and Ehlers (2007:14-25) argue that the entire health care system suffers because of the large number of nurses who emigrate, since nurses constitute the largest professional group in South Africa's health care system and form the backbone of PHC. Van Rensburg and Van Rensburg (1999:218) discuss strategies embarked upon by the South African Government to; address shortages, disparities and misdistribution of health professionals; recruitment of doctors from Cuba and other countries; the introduction of compulsory community service in 1998 for health professionals for two years after completing training; construction of residential accommodation in rural areas and rural allowance paid to attract health personnel; recruitment and bursaries for under-privileged individuals; and changes to the curriculum to attract and retain health professionals in the public service.

Communication and the demonstration of expert nursing care were perceived as positive when participants experienced a sensitive and caring attitude. Patients and their families judge nurses according to how they interact with others on the care team (Leebov 2009:1). A positive nurse-patient relationship enhances patient and family satisfaction. Attitudes of health care providers impacted on health care user care and may influence adherence with treatment plans. 
Participants were satisfied with shorter waiting times and associated longer waiting times with poorer service. Various authors like Thompson and Yarnold (1995), Eilers (2004), and Agaba, Bagul, Adenugba and Kenogbon (2002), agreed that patient satisfaction is directly linked to waiting time. Furthermore, the way organisations deal with clients whilst waiting impacts on the client's perception of the organisation. Queue management is about ensuring quality health care services and showing clients that they are waiting in a planned environment, whilst reassuring them that they will be attended to timeously.

Participants expressed satisfaction where the health care provider communicated with them, even if it was not health related. Communication by nurses has the greatest impact on how patients rate their experience of the service (Leebov 2009:1). Nurses are central in the care of patients; both patients and families rely on nurses to keep them informed and to connect them with the doctors and other health care providers, also to allay their anxiety during their health care experience. Yazdi, Tavafian, Emazdadeh, Kazemnejad and Ghofranipour (2008:140) found that the overall communication skills of health care providers improved patient satisfaction.

\section{Limitations of the study}

Data were collected from one geographic area within South Africa and, whilst a large number of participants were included, there was no opportunity to verify the responses with the participants. This exploratory study only sought the perceptions of the health care users of the Fast Queue; follow-up work should also gain insights from the health care providers.

\section{Recommendations}

The following recommendations emerged:

- Queue marshals could assist in directing users to respective queues to reduce waiting time and keep users satisfied when they feel that they are being taken care of.

- Use of clear sign posts where there is lack of human resources to direct users.

- Involvement of nurses in planning construction of health facilities to give input into what is required considering patient flow, infection prevention and control, and provision of privacy.

- Increasing financial support for nursing educational programmes that prepare the best educated nurses and focus on teaching effective health communication strategies.

- Effective complaints mechanism for users to ensure that complaints are followed up.

- Provision of employee assistance programmes and debriefing services to assist staff that have been reported to have less than desirable attitudes toward health care users.

- Gainfully occupying users whilst they are waiting like health promotion talks conducted by a person or watched on video.
- Encourage users to arrive at the facility throughout the day, rather than the present practice where all users arrive in the clinic early in the morning. This variable arrival rate (Fomundam \& Herrmann 2007:5) encourages users to arrive at times when the facility is less congested so that work is balanced throughout the day.

\section{Conclusion}

Overcrowding is a problem in health facilities and the Fast Queue is one strategy that is attempting to address it; however, it was demonstrated by the results of this study many factors contribute to the levels of satisfaction or dissatisfaction of users. It seems there is a need to improve on the different aspects of the Fast Queue, such as streamlining observations in an effort to address the problem of overcrowding and long waiting time in the health facilities. The involvement of the Healthy Ministry is crucial in supporting this endeavour, through building more spacious facilities and employment of more staff. However, even in settings with limited space, communication emerged as an important theme.

\section{Aknowledgements}

Thanks to the following people who contributed in many different ways to the research and writing of the manuscript: Mrs. J.N. Makhanya, N.M. Sibiya, Mrs. P.M. Orton. Prof. K. Nokes, who co-authored and edited the manuscript, I would also like to thank the reviewers of the manuscript in advance.

\section{Competing interests}

The authors declare that they have no financial or personal relationship(s) which may have inappropriately influenced them in writing this article.

\section{Authors' contributions}

D.G.S. (Durban University of Technology) wrote the manuscript. N.J.M. (Department of Health) was the principal supervisor of the study. N.M.S. (Durban University of Technology) was the co-supervisor of the study. K.N. (Durban University of Technology) co-authored and edited the manuscript.

\section{References}

Agaba, A.E., Bagul, A., Adenugba, J.B. \& Kenogbon J.I., 2002, 'Audit of patient's waiting time to see their family doctor prior to referral to a fast-access breast clinic in the era of a guaranteed 2-week wait', Breast 11(5), 430-433, viewed 23 March 2010 from http://www.ncbi.nlm.nih.gov/pubmed/14965707

Ajayi, I.O., 2002, 'Patients' waiting time at an outpatient clinic in Nigeria - can it be put to better use?' Patient Education and Counselling 47, 121-126, viewed 23 March 2010, from http://www.ncbi.nlm.nih.gov/pubmed/12191535

Barron, P., Day, C., Loveday, M. \& Monticelli, F., 2005, 'The District Health Barometer Year 1. January - December 2004', Health Systems Trust, viewed 23 March 2010, from http://www.hst.org.za/publications/689

Burns, N. \& Grove, S.K., 1999, The practice of nursing research: conduct, critique and utilization, 3rd edn., WB Saunders, Philadelphia.

CDC's HIV/AIDS Care and Treatment Programs in South Africa: TB and HIV, 2011, viewed 23 December 2012, from http://www.cdc.gov/globalhealth/countries/ southafrica/what/tb_hiv.htm

Department of Health, 2001, The comprehensive primary health care package for South Africa, Formeset Printers Cape for the Government Printer, Pretoria. 
Eilers, G.M., 2004, 'Improving patient satisfaction with waiting time', Journal of American College Health 53(1), 41-48, viewed 29 April 2010, from http://heldrefpublications.metapress.com/app/home/contribution.asp?referrer=parent\&backt o=issue, 6, 6; journal, 36, 78; linkingpublicationresults, 1:119928, 1

eThekwini Municipality Health Unit, n.d., Annual Report 2006/2007, Durban.

Fomundam, S. \& Herrmann, J., 2007, 'A survey of queuing theory applications in healthcare', [Online], ISR Technical Report 2007-24, viewed 23 March 2010, from http://www.lib.umd.edu/drum/bitstream/1903/7222/1/tr 2007-24.pdf

Hall, E. J., 2001, 'The challenges HIV/AIDS poses to nurses in their work environment', Employment and Economic Policy Research Human Sciences Research Council, viewed 17 March 2010, from http://www.hsrc.ac.za/research/ output/outputDocuments/3371_Hall_ThechallengesHIVposes.pdf

Kano, N., 1984, 'Customer Satisfaction Model', in 12 manage, viewed 23 December 2012, from http://www.12manage.com/methods_kano_customer_satisfaction_ model.html

Leatte, A., Shung-King, M. \& Monson, J., 2006, Healing inequalities: the free health policy [online], Children's Institute, University of Cape Town, Cape Town. viewed 17 March 2010, from http://www.ci.org.za/depts/ci/pubs/pdf/general/ gauge2006/gauge2006_healing.pdf

Leebov, W., 2009, 'The quality experience: the nurse patient relationship is central to patient satisfaction', in The quality patient experience, viewed 16 February 2010 ,
from http://www.quality-patient-experience.com/nurse-patient-relationship.html

Mahlangu, Q., 2009, Health Budget speech tabled by MEC for Health and Social Development, Ms Qedani Dorothy Mahlangu', in South African Government information, viewed 17 March 2010, from http://www.info.gov.za/speeches/ 2009/09080614251002.htm

Oakland, J.S., 2003, Total quality management: text with cases, ButterworthHeinemann, Oxford.

Oosthuizen, M. \& Ehlers V.J., 2007, 'Factors that may influence South African nurses' decisions to emigrate', Health SA Gesondheid 12(2), 14-26. http://dx.doi. org/10.4102/hsag.v12i2.246
Pendukeni, M., 2004, 'The impact of HIV/AIDS on health care provision: Perceptions of nurses currently working in one regional hospital in Namibia', Master's in Public Health mini-thesis, [online], University of the Western Cape, viewed 26 February 2010, from http://etd.uwc.ac.za/usrfiles/modules/etd/docs/etd 26 February 2010, from
init_3642_1175160167.pdf

Qmatic.com, 2005, Queuing management system, viewed 23 December 2012, from http://www.qmatic.com/en/Sectors/Healthcare/

Setty, V., 2004, Organizing work better, HRH Global Resource Center, viewed 26 February 2010, from http://www.hrhresourcecenter.org/node/1076

Republic of South Africa, 2004, National Health Act (Act 61 of 2003), Government Printer, Pretoria.

Sibiya, M.N. 2009, 'A model for the integration of primary health care services in KwaZulu Natal, South Africa', D.Tech thesis, Nursing in the Faculty of Health Sciences, Durban University of Technology, viewed 26 February 2010, from http:// ir.dut.ac.za/xmlui/handle/10321/453

Thompson, D.A. \& Yarnold, P.R., 1995, 'Relating Patient satisfaction to waiting time perception and expectations: The disconfirmation paradigm', Academic Emergency Medicine, 2(12), 1057-1062, viewed 23 March 2010, from http://www3. interscience.wiley.com/journal/121422600/abstract?CRETRY $=1 \&$ SRETRY $=0$

Van Rensburg, D. \& Van Rensburg, N., 1999, 'Distribution of human resources', in N. Crisp, \& A. Ntuli, (eds.), South African Health Review 1999, Health Systems Trust, Durban. PMCid: 1734236

World Health Organisation (WHO), 1978, Declaration of Alma-Ata, International Conference on Primary Health Care, Alma Ata, USSR, WHO, Geneva, 06-12 September 1978, viewed 23 March 2010, from http://www.who.int/hpr/NPH/ docs/declaration_almaata.pdf

Yazdi, N.A., Tavafian S.S., Emadzadeh, A., Kazemnejad, A. \& Ghofranipour, F., 2008 'Communication training and patient satisfaction: A randomized trial study from Mashhad, Iran', Patient Preference and Adherence 2(2), 137-142, viewed 23 March 2010, from http://www.ncbi.nlm.nih.gov/pubmed/19920954 